such as presence of $\mathrm{T}_{\mathrm{H}} 2$ cytokines IL-4 or IL-13 in vitro (Martinenaite et al, 2019, DOI: 10.1038/s41423-019-0231-3 and DOI: 10.1007/s00262-019-02425-6).

Methods and Results In order to explore if arginase-1-specific $\mathrm{T}$ cells have a potential role in modulation of immune homeostasis, human arginase-1-specific memory $\mathrm{T}$ cells were isolated and expanded for functional characterization. We show that arginase-1-specific $\mathrm{T}$ cells specifically recognize arginase-1 expressing cells, such as mRNA transfected autologous dendritic cells (DCs) and B cells as well as M2 polarized macrophages in vitro. In addition, activated arginase-1-specific $\mathrm{T}$ cells produce pro-inflammatory cytokines IFN $\gamma$ and TNF $\alpha$. Secretion of TH1 cytokines by these T cells suggests potential role as potent immune modulators in the tumor microenvironment, since many arginase- 1 expressing myeloid cells are not terminally differentiated and they can be re-polarized to an immunostimulatory, M1-like phenotype. We also observed that targeting of M2-polarized arginase-1 expressing monocytic leukemia cell line THP-1 with arginase-1-specific CD4+ T cells induces upregulation of PD-L1 on the THP-1 cells. Furthermore, we demonstrate that an arginase-1-derive peptide vaccine has a therapeutic effect in syngeneic mouse tumor models (B16 and MC38), both as monotherapy and in combination with anti-PD-1 treatment. The therapeutic effect was associated with increased immune infiltration in the peptide vaccinated mice compared to the control.

Conclusions Our study provides evidence that immune modulatory vaccination targeting arginase- 1 is an intriguing way of targeting the immune suppressive microenvironment.

Disclosure Information E. Martinenaite: A. Employment (full or part-time); Significant; IO Biotech. M. Aaboe Joergensen: None. R.E. Johansson Mortensen: None. S. Munir Ahmad: None. S.E. Weis-Banke: None. M. Orebo Holmström: None. A. Wakatsuki Pedersen: A. Employment (full or part-time); Significant; IO Biotech. Ö. Met: None. I.M. Svane: F. Consultant/Advisory Board; Significant; IO Biotech. M. Hald Andersen: A. Employment (full or part-time); Significant; IO Biotech.

\section{P04.06 MUCOSAL IMMUNIZATION WITH A CDC1-TARGETED CTA1 ADJUVANT VACCINE CONFERS PROTECTION AGAINST MELANOMA METASTASIS}

\begin{abstract}
${ }^{1} \mathrm{M}$ Arabpour*, ${ }^{1} \mathrm{~S}$ Paul, ${ }^{1} \mathrm{R}$ Kiffin, ${ }^{1} \mathrm{HG}$ Wiktorin, ${ }^{1} \mathrm{~K}$ Hellstrand, ${ }^{2} \mathrm{~N}$ Lycke, ${ }^{1} \mathrm{~A}$ Martner. ${ }^{1}$ TIMM Laboratory, Sahlgrenska Cancer Center, Institute of Biomedicine, Sahlgrenska Academy, University of Gothenburg, Göteborg, Sweden; ${ }^{2}$ Mucosal Immunobiology and Vaccine Center (MIVAC), Department of Microbiology and Immunology, Institute of Biomedicine, University of Gothenburg, Göteborg, Sweden
\end{abstract}

\subsection{6/jitc-2020-ITOC7.75}

Background Specific targeting of anti-cancer vaccines to dendritic cells (DCs) has been shown to mount efficient immune responses against tumor cells. Classical $\mathrm{CD} 103^{+}$dendritic cells (also called cDC1) have an inherent ability to cross-present antigens to $\mathrm{CD}^{+}$cytotoxic $\mathrm{T}$ cells. Here we have explored an anti-tumor vaccine that specifically targets cDC1 cells for protection against and elimination of metastatic melanoma. The vaccine contains the cholera toxin A1 subunit (CTA1) adjuvant and is targeted to cDC1 cells through an anti-CD103 single chain antibody (CD103 scFv).

Material and Methods C57BL/6 mice were injected with wild type or ovalbumin (OVA) expressing B16 melanoma cells either subcutaneously (s.c.) to establish solid tumors, or intravenously (i.v.) to allow the formation of pulmonary metastases. Before or after establishment of tumors, mice were intranasally inoculated with a vaccine composed of a CD103 scFv element fused to the adjuvant CTA1 and the MHC I H2kdrestricted OVA epitope SIINFEKL together with the MHC II H2kd-restricted OVA epitope p323 or just the p323 peptide alone (i.e. CTA1-SIINFEKL-p323-CD103 and CTA1-p323CD103, respectively). Control mice were inoculated with PBS. The growth of solid tumors was carefully monitored and the development of pulmonary metastases was determined 2-3 weeks after tumor cell injection. In addition, antigen-specific $\mathrm{T}$ cell immunity following intranasal immunization was evaluated.

Results Targeting MHC I and MHC II tumor cell epitopes to cDC1, via CD103 ScFv, in conjunction with the CTA1 adjuvant elicited strong tumor specific and protective $\mathrm{CD} 8^{+} \mathrm{T}$ cell responses as well as $\mathrm{CD}^{+} \mathrm{T}$ cell immunity. Immunization with the CTA1-SIINFEKL-p323-CD103 vaccine significantly reduced the growth of established solid B16F1-OVA melanomas $(P<0.001)$ and potently prevented metastasis formation $(P<0.01)$. Control immunizations with the CTA1-p323-CD103 vaccine tended to reduce metastasis, but tumor-specific CD8 ${ }^{+}$ $\mathrm{T}$ cells were required for full therapeutic protection.

Conclusion Targeting tumor specific $\mathrm{CD}^{+} \mathrm{T}$ cell epitopes to cDC1, in the context of a powerful adjuvant such as CTA1, leads to the development of efficient anti-tumor immune responses. Our results point towards the utility of cDC1-targeted vaccines in the treatment of established tumors or as a means to prevent metastasis formation.

Disclosure Information M. Arabpour: None. S. Paul: None. R. Kiffin: None. H.G. Wiktorin: None. K. Hellstrand: None. N. Lycke: None. A. Martner: None.

\section{P04.07 ABSTRACT WITHDRAWN}

\section{P04.08 VIRUS LIKE VACCINES: A NOVEL IMMUNOTHERAPY STRATEGY AGAINST THE CANCER-ASSOCIATED ENDOGENOUS RETROVIRUS}

1J Daradoumis*, ${ }^{2} \mathrm{PJ}$ Holst, ${ }^{2} \mathrm{KN}$ Nielsen. ${ }^{1}$ Copenhagen University, Copenhagen, Denmark; ${ }^{2}$ InProTher, Copenhagen, Denmark

10.1136/jitc-2020-ITOC7.76

In face of the necessity of broadly acting and highly effective vaccines capable of eliminating/preventing human cancers with insufficient mutated antigens, we introduced the concept of Virus-Like Vaccines (VLVs). This strategy combines a replication-deficient retrovirus encoding virus structural proteins. These proteins assemble into secreted viruslike particles (VLPs) that deliver the target antigen to the immune system rising both humoral and cellular immune responses. Here, we use an adenoviral vector encoding the group specific antigen (Gag) and the glycoprotein of the viral envelope (Env) from endogenous retrovirus (ERV). Since ERV Env is reported to have immunosuppressive properties that support tumor establishment and development, we designed a modified vaccine that includes a mutation on the Env immunosuppressive domain (ISD) that 